\title{
MACROPHAGE ACTIVATION SYNDROME (MAS) REFRACTORY TO IMMUNOGLOBULIN WITH EXCELLENT RESPONSE TO RITUXIMABE: CASE REPORT
}

Douglas Squizatto Leite ${ }^{1, *}$, Matheus Zanata Brufatto ${ }^{1}$, Sean Hideo Shirata Lanças ${ }^{1}$, Andrea de Almeida Peduti Batista ${ }^{1}$, Laura Maria Silva de Siqueira ${ }^{1}$, Henrique Pereira Sampaio ${ }^{1}$, Luiz Eduardo Valente ${ }^{1}$, Daniela Esteves Temporim ${ }^{1}$, João Flávio Gomes Faria $^{1}$, Sula Glaucia Lage Drumond Pacheco ${ }^{1}$

1. Universidade Estadual Paulista “Júlio de Mesquita Filho", Botucatu (SP), Brazil.

*Corresponding author: squizattoleite@gmail.com

\section{BACKGROUND}

Macrophage activation syndrome (MAS) is a rare condition, potentially fatal, characterized by massive production of proinflammatory cytokines that results in failure of multiple organs and can be triggered by an initial picture of ASD.

\section{CASE REPORT}

A male patient, 38 years old, previously healthy with a history of daily fever (above $39^{\circ} \mathrm{C}$ ), myalgia, odynophagia and dry cough for 3 weeks. He was admitted to our service prostrate, tachycardic, tachypneic, with cervical and inguinal lymphadenomegaly and erythematous cutaneous rash in the abdomen and upper chest. Pulmonary auscultation with bilaterally based crackles and presence of pericardial effusion on echocardiogram and hepatosplenomegaly on ultrasound. He presented significant leukocytosis without left deviation, increased inflammatory tests (ESR: 120). Initially entered with antibiotic and initial hypothesis of leukemoid reaction or adult still disease (DSA) was made. Hydrocortisone was started at a dose equivalent to $1 \mathrm{mg} / \mathrm{kg}$ prednisone and an inguinal LFN biopsy was requested to rule out malignancy (result compatible with reactive lymphadenitis). He evolved with acute kidney injury requiring dialysis support. On the 5th day of hospitalization, he presented an increase in liver enzymes, triglycerides and ferritin > 2000, as well as a decrease in fibrinogen and ESR, with hemophagocytosis confirmed in the MO aspirate. Diagnosis of MAS was made and pulse of methylprednisolone ( $1 \mathrm{~g}$ for 3 days) and intravenous immunoglobulin ( $2 \mathrm{~g} / \mathrm{kg}$ divided in 5 days) were performed. After initial improvement, on the 13th day of hospitalization, he evolved with cardiac arrest, ventricular fibrillation, being reversed after 5 cycles. In the post-CPR, cardiac tamponade was found, puncture was performed with exudative fluid and it was decided to expand antibiotic therapy. After extubation, he developed fever (negative cultures) and opted for rituximab. He presented gradual clinical and laboratory improvement and hospital discharge, maintaining remission and complete resolution of the MAS.

\section{CONCLUSION}

Report of a severe case of MAS, with cardiac tamponade and multiple systemic manifestations, refractory to pulse therapy with corticosteroids and immunoglobulin and with an excellent response to rituximab, progressing with sustained remission.

\section{KEYWORDS}

Macrophage activation syndrome, Rituximab, Cardiac tamponade. 